\title{
Secretory expression and characterization of a highly Ca2+-activated thermostable L2 lipase
}

\begin{abstract}
Thermostable lipases are important biocatalysts, showing many interesting properties with industrial applications. Previously, a thermophilic Bacillus sp. strain L2 that produces a thermostable lipase was isolated. In this study, the gene encoding for mature thermostable L2 lipase was cloned into a Pichia pastoris expression vector. Under the control of the methanolinducible alcohol oxidase (AOX) promoter, the recombinant L2 lipase was secreted into the culture medium driven by the Saccharomyces cerevisiae Ŭfactor signal sequence. After optimization the maximum recombinant lipase activity achieved in shake flasks was 125 $\mathrm{U} / \mathrm{ml}$. The recombinant $44.5 \mathrm{kDa}$ L2 lipase was purified 1.8-fold using affinity chromatography with $63.2 \%$ yield and a specific activity of $458.1 \mathrm{U} / \mathrm{mg}$. Its activity was maximal at $70{ }^{\circ} \mathrm{C}$ and $\mathrm{pH}$ 8.0. Lipase activity increased 5-fold in the presence of Ca2+. L2 lipase showed a preference for medium to long chain triacylglycerols (C10ї C16), corn oil, olive oil, soybean oil, and palm oil. Stabilization at high temperature and alkaline $\mathrm{pH}$ as well as its broad substrate specificity offer great potential for application in various industries that require high temperature operations.
\end{abstract}

Keyword: Recombinant thermostable lipase; Pichia pastoris; $\mathrm{Ca} 2+$ activation; High yield purification; Recombinant lipase; Thermostable 